\title{
Dúvidas na gestação: vivência de mulheres acompanhadas na atenção primária à saúde
} Pregnancy questions: the experience of women accompanied in primary health care

\section{Dudas sobre el embarazo: experiencia de mujeres atendidas en la atención primaria a la salud}

\author{
Recebido: $17 / 09 / 2020$ \\ Aprovado: 04/03/2021 \\ Publicado: 14/10/2021
}

\section{Beatriz Lisboa de Macedo Brito ${ }^{1}$ Bianca Machado Cruz Shibukawa² Maria de Fátima Garcia Lopes Merino $^{3}$ Ieda Harumi Higarashi ${ }^{4}$ Marcela Demitto Furtado 5}

Trata-se de um estudo transversal, descritivo, exploratório com abordagem quantitativa, realizado entre fevereiro e junho de 2019, com 33 gestantes pertencentes à área de abrangência de uma Unidade Básica de Saúde localizada na região noroeste do Paraná, com o objetivo de identificar as principais dúvidas vivenciadas na gestação por mulheres acompanhadas na atenção primária à saúde. Utilizou-se questionário semiestruturado e entrevista e os dados foram analisados mediante estatística descritiva. A idade estava entre 19 e 35 anos (78,79\%), raça/cor não branca (54,55\%), nove anos ou mais de estudo (87,88\%), com companheiro (93,94\%), ocupação profissional remunerada (57,58\%), renda igual ou maior que três salários mínimos (72,72\%), e número de pessoas no mesmo domicílio inferior ou igual a cinco $(87,88 \%)$. Duas ou mais gestações anteriores $(75,76 \%)$, parto normal $(42,42 \%)$, gravidez atual sem planejamento $(63,64 \%)$, no segundo trimestre (51,52\%); com risco gestacional baixo e alto risco $(42,42 \%$ cada). Das entrevistadas, 61\% relataram dúvidas categorizadas em: Alterações fisiológicas; Parto e nascimento; Amamentação; Cuidados com o recém-nascido; e, Outras. Nas redes de apoio tem-se: a mãe, a internet e a Unidade Básica de Saúde. Em $52 \%$ das gestantes houve sugestões de mudanças, com especial referência aos profissionais da UBS e a forma de condução das consultas de pré-natal. Assim, o profissional de saúde deve acompanhar as gestantes de forma compreensível, vendo-a como um todo, para esclarecer medos e ansiedades, buscando proporcionar uma experiência positiva e segura da gestação.

Descritores: Gravidez; Cuidado pré-natal; Educação em saúde; Atenção primária à saúde.

This is a cross-sectional, descriptive, exploratory study with a quantitative approach, carried out between February and June 2019, with 33 pregnant women in the coverage area of a Basic Health Unit located in the Northwestern region of the state of Paraná, Brazil. It aimed to identify the main doubts experienced during pregnancy by women being cared for in primary health care. A semi-structured questionnaire and interview were used; data were analyzed using descriptive statistics. Age was between 19 and 35 years (78.79\%), non-white race/color (54.55\%), nine years or more of education (87.88\%), with a partner (93.94\%), occupation paid professional $(57.58 \%)$, income equal to or greater than three minimum wages $(72.72 \%)$ and a number of people living in the same household less than or equal to five (87.88\%). Two or more previous pregnancies (75.76\%), vaginal delivery (42.42\%), current pregnancy being unplanned (63.64\%), being in the second trimester (51.52\%); with low and high gestational risk ( $42.42 \%$ each). Of the interviewees, $61 \%$ reported doubts categorized as: Physiological alterations; Delivery and childbirth; Breastfeeding; Newborn care; and Others. In support networks, there are: the mother, the internet and the Basic Health Unit (UBS). In 52\% of the pregnant women there were suggestions for changes, with special reference to UBS professionals and the way in which prenatal consultations were conducted. Thus, the health professional must follow the pregnant women in an understandable way, seeing them as a whole, to clarify fears and anxieties, seeking to provide a positive and safe experience of pregnancy.

Descriptors: Pregnancy; Prenatal care; Health education; Primary health care.

Se trata de un estudio transversal, descriptivo y exploratorio con enfoque cuantitativo, realizado entre febrero y junio de 2019, con 33 embarazadas pertenecientes al área de cobertura de una Unidad Básica de Salud ubicada en la región noroeste de Paraná, Brasil, con el objetivo de identificar las principales dudas vividas en el embarazo por mujeres acompañadas en la atención primaria a la salud. Se utilizó un cuestionario semiestructurado y una entrevista; los datos se analizaron mediante una estadística descriptiva. La edad estaba entre 19 y 35 años (78,79\%), raza/color no blanco $(54,55 \%)$, nueve años o más de estudio $(87,88 \%)$, con pareja $(93,94 \%)$, ocupación profesional remunerada $(57,58 \%)$, ingresos iguales o superiores a tres salarios mínimos $(72,72 \%)$ y número de personas en el mismo hogar menor o igual a cinco $(87,88 \%)$. Dos o más embarazos previos $(75,76 \%)$, parto normal $(42,42 \%)$, embarazo actual no planeado $(63,64 \%)$, en el segundo trimestre (51,52\%); con riesgo gestacional bajo y alto $(42,42 \%$ cada uno). De las entrevistadas, el 61\% declaró dudas clasificadas como: Cambios fisiológicos; Parto y nacimiento; Lactancia; Cuidados del recién nacido; y Otras. Las redes de apoyo fueron: la madre, Internet y la Unidad Básica de Salud. En el 52\% de las embarazadas hubo sugerencias de cambio, con especial referencia a los profesionales de la UBS y a la forma de realizar las consultas prenatales. Así, el profesional de la salud debe acompañar a las mujeres embarazadas de forma comprensible, viéndolas como un todo, para aclarar temores y ansiedades, buscando proporcionar una experiencia positiva y segura de embarazo.

Descriptores: Embarazo; Atención prenatal; Educación en salud; Atención primaria de salud.

1. Enfermeira. Maringá, PR, Brasil. ORCID: 0000-0002-9666-7111 E-mail: beatrizbrito@gmail.com

2. Enfermeira. Especialista em Gestão de Pessoas. Mestre em Enfermagem. Doutoranda no Programa de Pós Graduação em Enfermagem (P PGE) pela Universidade Estadual de Maringá (UEM), Maringá, PR, Brasil ORCID: 0000-0002-7739-7881 E-mail: bih.cruuz@gmail.com

3. Enfermeira. Historiadora. Especialista em Docência na Saúde. Mestre e Doutora em Enfermagem. Professora do curso de Graduação em Enfermagem da UEM, Maringá, PR, Brasil. ORCID: 0000-0001-6483-7625 E-mail: fatimamerino@gmail.com

4. Enfermeira. Especialista em Enfermagem Pediátrica e Puericultura. Mestre em Educação Especial. Doutora em Educação. Professora do PPGE-UEM, Maringá, PR, Brasil. ORCID: 0000-0002-4205-6841 E-mail: ieda1618@gmail.com

5. Enfermeira. Especialista em Enfermagem Obstétrica. Mestre e Doutora em Enfermagem. Docente do curso de Graduação em Enfermagem da UEM, Maringá, PR, Brasil. ORCID: 0000-0003-1427-4478 E-mail: mar_demitto@hotmail.com 


\section{INTRODUÇÃO}

1 gravidez é um momento almejado e esperado pela maioria das mulheres, muito complexo e único, seguido de mudanças contínuas que abrangem todas as áreas da vida,

afetando-a de forma biopsicossocial. A gestante necessita de cuidados que atinjam esses diversos aspectos, que vão desde o autoconhecimento, o amparo familiar, a relação com o companheiro, até o tratamento que recebe pelos profissionais da saúde ${ }^{1}$.

Para se refletir sobre a diligência que a gestante requer, é preciso entender que o modo como a gestação é vista e tratada difere entre uma mulher e outra. Esta diversificação também reside no aspecto temporal, pois uma mesma pessoa pode reagir de modo diferente a este evento em seu corpo no transcorrer dos anos².

Desde a descoberta da gravidez até o momento do nascimento, a mulher vivencia muitas dúvidas e preocupações. No primeiro trimestre, estas estão voltadas para as mudanças com o corpo, emoções e à habituação da nova fase. No segundo trimestre, a partir das primeiras movimentações do feto, a gestante se concentra na idealização de alguém se desenvolvendo em seu interior. Por sua vez, no terceiro trimestre, o foco está direcionado para o tipo de parto, o momento do nascimento e cuidados posteriores ${ }^{2,3}$.

É comum a presença de dúvidas no período gestacional acerca de diversos assuntos. Elas vão desde as mudanças físicas e desenvolvimento do feto, até o tipo de parto e cuidados no período pós-natal. No entanto, ainda há lacunas no conhecimento científico sobre os principais questionamentos das mulheres nesse período da vida, o que implica em aumento da ansiedade, medos e angústias, o que pode provocar riscos à saúde da mãe e do bebê. Os questionamentos das gestantes precisam ser respondidos com vistas a proporcionar segurança e confiança, tanto no período gravídico, quanto no puerperal ${ }^{4}$.

Para tal, a gestante busca fontes de apoio e auxílio, como a família e os serviços de saúde. Ante a esta demanda, a Unidade Básica de Saúde (UBS) é responsável pela realização do prénatal, tornando-se um espaço no qual os profissionais exercem importante papel no acompanhamento e esclarecimento de dúvidas dessas mulheres, à medida que acolhem e oferecem informações relevantes para uma gestação de qualidade ${ }^{3,5}$.

Compreender as dúvidas das gestantes pode auxiliar os profissionais no planejamento direcionado de atividades de educação em saúde, proporcionando a compreensão do pré-natal como um processo coletivo e de compartilhamento de responsabilidades, além de buscar ofertar por meio da informação, uma experiência positiva da gestação ${ }^{2,6}$. Nesse sentido, o presente estudo teve como objetivo identificar as principais dúvidas vivenciadas na gestação por mulheres acompanhadas na atenção primária à saúde.

\section{MÉTODO}

Trata-se de um estudo transversal, descritivo, exploratório com abordagem quantitativa, realizado com gestantes pertencentes à área de abrangência de uma UBS localizada no noroeste do Paraná; e que estavam cadastradas no sistema eletrônico, denominado "Gestor Saúde", no mês de fevereiro de 2019.

A referida UBS possui duas equipes da Estratégia Saúde da Família (ESF). Ambas possuem: enfermeiro, técnico de enfermagem, médico e agentes comunitários de saúde, e atualmente atendem aproximadamente 7.500 pessoas. A UBS recebe o apoio de médicos residentes da área de ginecologia e obstetrícia para o acompanhamento das gestantes de risco intermediário, bem como as de alto risco.

Primeiramente, foi realizada uma busca pelas gestantes cadastradas no sistema eletrônico. De posse desta lista, obteve-se o nome, endereço, telefone de contato e a idade gestacional que as grávidas se encontravam. As gestantes foram então contactadas via telefone, por meio do qual foi explicada a proposta da pesquisa; após o aceite, um horário para a entrevista foi agendado. Considerou-se até três tentativas realizadas em dias e horários 
distintos. As entrevistas foram realizadas na data agendada em local reservado para garantir o conforto e sigilo das entrevistadas.

Os dados foram coletados entre os meses de fevereiro e junho de 2019 por meio de um instrumento semiestruturado, elaborado pelos pesquisadores. Tal ferramenta de investigação possuía questões sobre o perfil sociodemográfico das gestantes, história obstétrica atual, dúvidas vivenciadas na gestação e possíveis redes de apoio.

Os dados foram transcritos para uma planilha eletrônica no programa Microsoft Excel@ e analisados por meio de estatística descritiva.

O estudo respeitou as Diretrizes e Normas Regulamentadoras de Pesquisas Envolvendo Seres Humanos do Conselho Nacional de Saúde (Resolução CNS 466/2012) e foi aprovado conforme CAAE: 96376318.6.0000.0104 e Parecer $\mathrm{n}^{\circ}$ 3.073.257/2018 do Comitê Permanente de Ética em Pesquisa com Seres Humanos da Universidade Estadual de Maringá (COPEP).

\section{RESULTADOS}

O levantamento inicial identificou 53 gestantes elegíveis, porém participaram 33, visto que no período da coleta, cinco já haviam parido, sete mudaram de endereço e oito não atenderam o contato telefônico.

A maioria possuía idade entre 19 e 35 anos (78,79\%), raça/cor não branca (54,55\%), nove anos ou mais de estudo (87,88\%), com companheiro $(93,94 \%)$, ocupação profissional remunerada $(57,58 \%)$, renda igual ou maior que três salários mínimos $(72,72 \%)$, e número de pessoas no mesmo domicílio inferior ou igual a cinco $(87,88 \%)$ (Tabela 1$)$.

No histórico obstétrico já possuía duas ou mais gestações anteriores $(75,76 \%)$, com destaque para o parto normal $(42,42 \%)$. Na gravidez atual, não planejou engravidar $(63,64 \%)$, estava no segundo trimestre (51,52\%); e sobre a classificação de risco gestacional, o mesmo número de mulheres apresentou baixo e alto risco (42,42\% cada) (Tabela 1$)$.

Das entrevistadas, $61 \%$ relataram dúvidas vivenciadas no período gestacional, as quais foram categorizadas para melhor compreensão em: Alterações fisiológicas; Parto e nascimento; Amamentação; Cuidados com o recém-nascido; e, Outras (Tabela 2).

Das gestantes que relataram dúvidas, 12 (60\%) expuseram incertezas em apenas uma das áreas; seis delas (30\%) em duas áreas; uma (5\%) em três áreas; e uma (5\%) em quatro. Considerando o período de gravidez que elas se encontravam no momento da coleta de dados, observou-se que $50 \%$ das gestantes do primeiro trimestre relataram dúvidas; bem como $60 \%$ do segundo trimestre e $64 \%$ do terceiro trimestre (Figura 1).

Com exceção de duas, todas as gestantes entrevistadas demonstraram possuir redes de apoio que as auxiliavam na vivência das dúvidas. Dentre as redes que mais se destacaram temse: a mãe da gestante (22\%), a internet (22\%), UBS (22\%), leituras (10\%), outros familiares (8\%) e planos particulares de saúde (7\%).

Acerca de sugestões de mudanças para melhorar a experiência da gestação em relação à presença de dúvidas nesse período, em $52 \%$ se recomendou algo, fazendo referência aos profissionais da UBS e a forma de condução das consultas de pré-natal. Nesse sentido, as mulheres propuseram que a consulta fosse mais acolhedora e com mais aconselhamento empático. Também recomendaram: grupos de discussão, treinamentos, exercícios de preparação para o parto e diminuição da rotatividade dos profissionais, aumentando o vínculo e a troca de saberes. 
Tabela 1. Perfil sociodemográfico e obstétrico das gestantes participantes do estudo. Maringá, Paraná, 2019.

\begin{tabular}{|c|c|c|}
\hline Perfil sociodemográfico & $\mathrm{N}$ & $\%$ \\
\hline \multicolumn{3}{|l|}{ Idade materna } \\
\hline $19-35$ & 26 & 78,79 \\
\hline$\geq 36$ & 7 & 21,21 \\
\hline \multicolumn{3}{|l|}{ Raça/cor } \\
\hline Branca & 15 & 45,45 \\
\hline Não branca & 18 & 54,55 \\
\hline \multicolumn{3}{|l|}{ Escolaridade } \\
\hline$\leq 4$ anos & 1 & 3,03 \\
\hline $5-8$ anos & 3 & 9,09 \\
\hline$\geq 9$ anos & 29 & 87,88 \\
\hline \multicolumn{3}{|l|}{ Situação conjugal } \\
\hline Com companheiro & 31 & 93,94 \\
\hline Sem companheiro & 2 & 6,06 \\
\hline \multicolumn{3}{|l|}{ Ocupação profissional } \\
\hline Não remunerada & 14 & 42,42 \\
\hline Remunerada & 19 & 57,58 \\
\hline \multicolumn{3}{|l|}{ Renda familiar } \\
\hline$\leq 2$ salários mínimos & 7 & 21,21 \\
\hline$\geq 3$ salários mínimos & 24 & 72,72 \\
\hline Não sabe & 2 & 6,07 \\
\hline \multicolumn{3}{|l|}{ № de moradores } \\
\hline$\leq 5$ & 29 & 87,88 \\
\hline$\geq 6$ & 4 & 12,12 \\
\hline \multicolumn{3}{|l|}{ Histórico obstétrico } \\
\hline \multicolumn{3}{|l|}{ № de gestações } \\
\hline 1 & 8 & 24,24 \\
\hline$\geq 2$ & 25 & 75,76 \\
\hline \multicolumn{3}{|l|}{ Partos anteriores } \\
\hline Normal & 14 & 42,42 \\
\hline Cesárea & 11 & 33,33 \\
\hline Não se aplica & 8 & 24,24 \\
\hline \multicolumn{3}{|l|}{ Gravidez planejada } \\
\hline Sim & 12 & 36,36 \\
\hline Não & 21 & 63,64 \\
\hline \multicolumn{3}{|l|}{ Idade gestacional } \\
\hline $1^{\circ}$ trimestre & 4 & 12,12 \\
\hline $2^{\circ}$ trimestre & 17 & 51,52 \\
\hline 3o trimestre & 12 & 36,36 \\
\hline \multicolumn{3}{|l|}{ Classificação de risco } \\
\hline Baixo & 14 & 42,42 \\
\hline Intermediário & 5 & 15,15 \\
\hline Alto & 14 & 42,42 \\
\hline
\end{tabular}


Tabela 2. Dúvidas vivenciadas na gestação por mulheres acompanhadas na atenção primária à saúde. Maringá, Paraná, 2019.

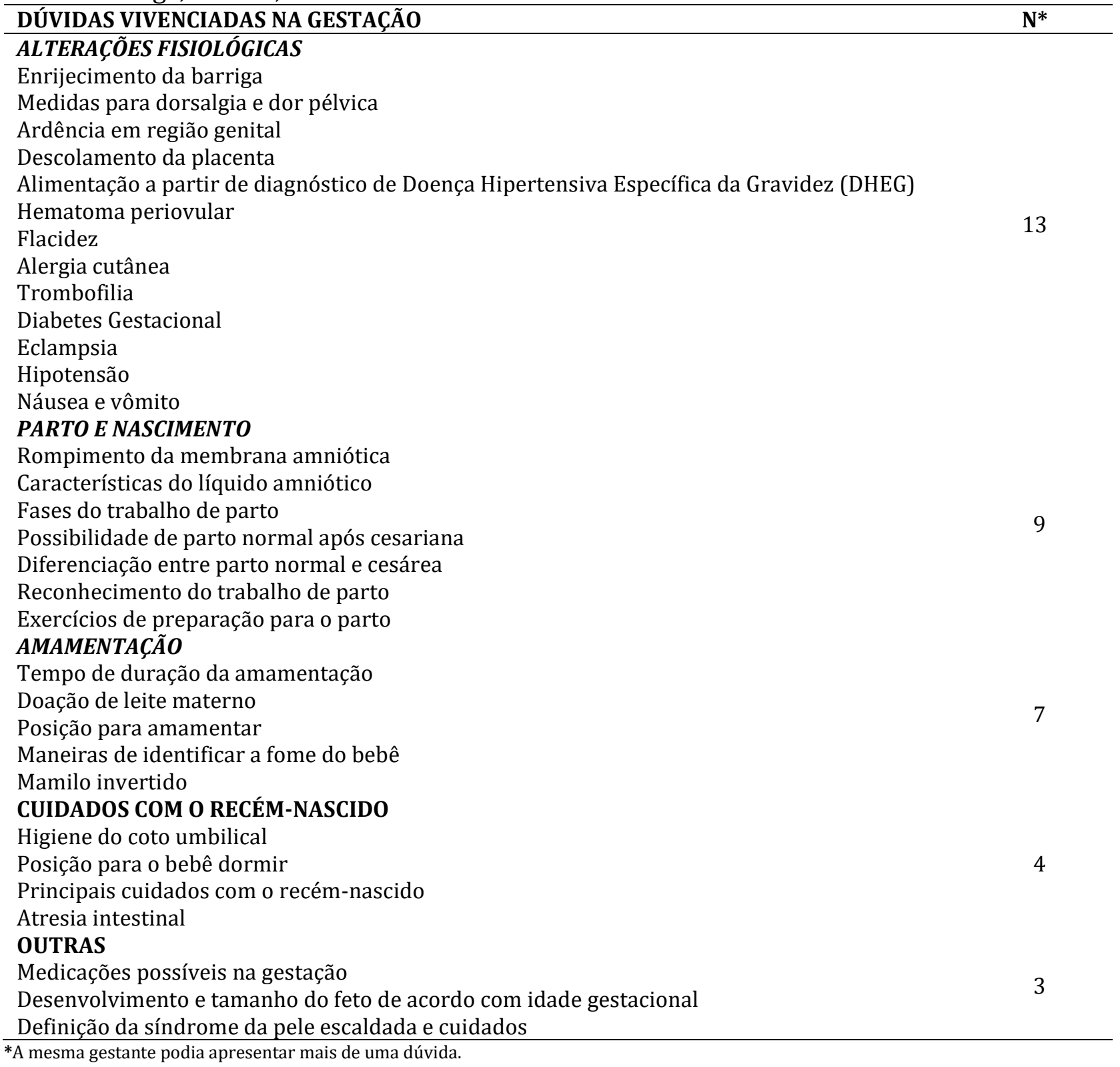

Figura 1. Classificação das gestantes de acordo com o trimestre gestacional e sua relação com a presença de dúvidas. Maringá, Paraná, 2019.

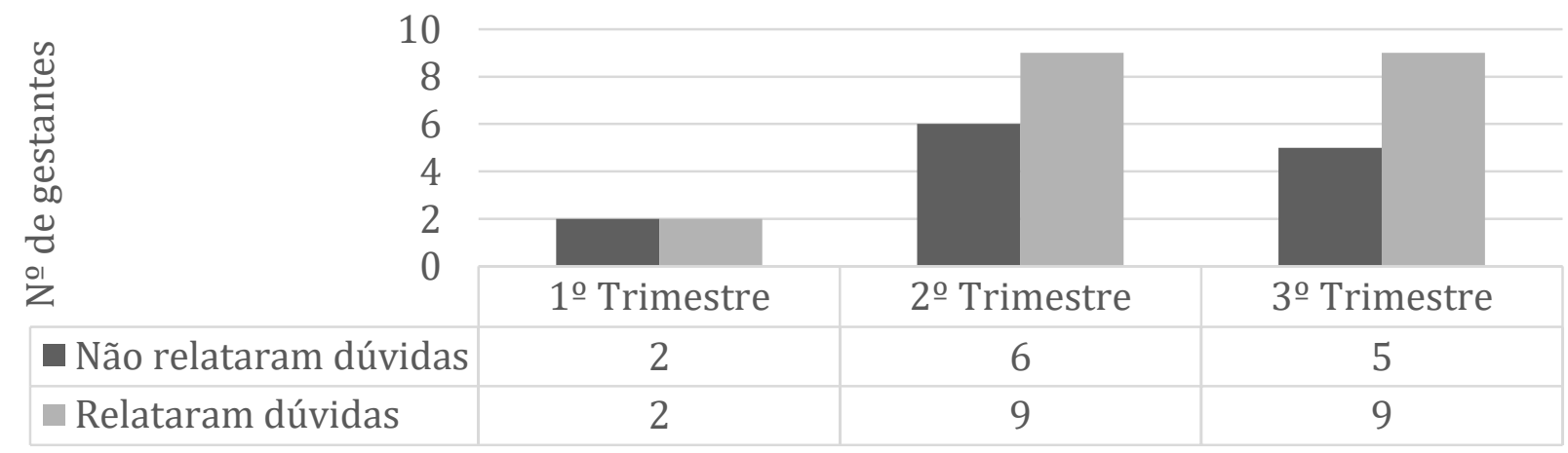




\section{DISCUSSÃO}

A gravidez é um período que gera mudanças em todas as áreas da vida da mulher, acompanhada de singularidades emocionais e sociais, com vulnerabilidades que desencadeiam dúvidas. 0 receio de uma gravidez malsucedida torna a mulher suscetível e desejosa de conhecimento. Em consequência disto, as incertezas podem ser convertidas em maior receptividade dos profissionais da saúde, familiares e amigos que podem ajudar e oferecer informações. Logo, prover o fortalecimento do vínculo de confiança e da troca de experiência ${ }^{3,4}$.

A dúvida transforma-se num mecanismo mental da procura do saber, um senso investigativo que se estabelece no indivíduo que pensa. Isso ocorre com a gestante quando confrontada pelas inúmeras informações que recebe, com desconfiança do que ouve, ou desenvolvendo atitude crítica, o que pode gerar o crescimento e a autossuperação ${ }^{6}$.

Sobre o conteúdo das dúvidas em pesquisa realizada com oficinas educativas, em um Centro de Saúde da Família, em Sobral, Ceará, se identificou: aleitamento materno e no período gestacional como um todo ${ }^{7}$. Em outros trabalhos as dúvidas estavam relacionadas às alterações fisiológicas, à amamentação e à análise do conhecimento e expectativas de gestantes a respeito do parto e seus procedimentos ${ }^{8-11}$.

As alterações fisiológicas que ocorrem com a mulher, desde a fecundação até o pós-parto, a partir de modificações do metabolismo, do sistema imunológico e nos hormônios são comuns. É importante que as mulheres sejam orientadas acerca de que todos os sistemas corporais são afetados, para se promover nutrição, acolhimento do feto, preparação para o parto e amamentação ${ }^{12}$.

Também é importante o conhecimento acerca das diferenças entre alterações em uma gestação sem complicações daquela que apresenta disfunções patológicas, para assim adquirirem um saber mais acurado ${ }^{13}$.

Dentre os assuntos de interesse das gestantes, a temática de aleitamento materno sempre causa receio. Porém, uma pesquisa vinculada à Universidade do Estado do Rio de Janeiro (UERJ), evidenciou que já há maior duração do aleitamento materno nas últimas duas décadas, comparado ao período de 1970. Este êxito fora influenciado pelas novas políticas de saúde e a implantação de atividades visando a promoção e apoio à amamentação introduzidas na década de $1980^{14}$.

Entretanto, apesar da taxa de cobertura de pré-natal brasileira ser de cerca de $80 \% 15$, constatou-se que menos de $10 \%$ das mulheres grávidas foram orientadas no pré-natal a respeito do aleitamento materno e do parto ${ }^{16}$. Fato esse que se explica, em parte, pela falta de regularidade e padrão efetivo nas ações da atenção primária, além da carência de quantidade e qualidade das orientações realizadas. Estes dados são preocupantes e advertem a necessidade de reformulação e planejamento no pré-natal ${ }^{15}$.

Uma meta-análise realizada para identificar os fatores associados ao início e à continuidade da amamentação destacou, entre outros fatores, que a educação materna sobre o aleitamento materno interfere significativamente. Tal fato mostra a importância de intervenções direcionadas à educação e apoio de mulheres e suas famílias para uma amamentação bem-sucedida ${ }^{17}$.

De todas as imprecisões da gestação, o parto é a situação mais antagônica por causa da alegria e da imprevisibilidade. Ademais, as mulheres são constantemente influenciadas por testemunhos familiares, ações da mídia, experiências anteriores, incerteza quanto à habilidade para ser ou continuar sendo uma boa mãe e o temor de sentirem dor demasiada. Contudo, as gestantes que recebem orientações apropriadas demonstram ansiedade reduzida, colaboram e interagem mais com os profissionais e vivem uma experiência mais satisfatória ${ }^{18}$.

O papel dos profissionais que atuam na atenção primária é fundamental, pois são eles os responsáveis imediatos por acolher as gestantes, bem como por fornecer o preparo e o conhecimento necessários para as futuras mães enfrentarem o nascimento do seu bebê ${ }^{19}$. 
Outra dúvida é o cuidado com o recém-nascido, por conta de o bebê apresentar várias fragilidades. A relação do medo com a falta de preparo pode produzir falhas na diligência para com o filho $(\mathrm{a})^{20}$. As dúvidas quanto ao zelo com a criança recém-nascida podem estar pautadas aos mitos existentes entre as pessoas com as quais essas mulheres convivem. A influência das crendices e o saber popular se tornam uma adversidade para o discernimento entre o certo e o errado, sendo capaz tanto de contribuir como de dificultar os cuidados. Os profissionais de saúde devem procurar entender a cultura, identificar as dúvidas, as concepções, as experiências, as apreensões e as expectativas da gestante, sem querer comunicar uma mera transferência de dados e informações ${ }^{16,21}$.

0 fato das mulheres que relataram dúvidas estarem em trimestres gestacionais diferentes confirma que os questionamentos estão presentes em todas as etapas da gestação, e cada uma delas possui incertezas mais predominantes. Da mesma forma, as dúvidas foram vivenciadas tanto por primigestas como por multigestas, levando à assimilação de que cada gestação significa uma nova e única experiência, com características particulares e inusitadas ${ }^{1,4}$.

0 trabalho refutou a ideia de senso comum expresso pelo pensamento de que, a partir de uma vivência materna anterior as gestações seguintes, seriam mais "fáceis". As primigestas, normalmente, temem a falta de experiência nesta nova fase da vida; já as multigestas sofrem com as inúmeras cobranças, receio de sofrerem em alguns aspectos desagradáveis vividos em gravidez(es) anterior(es), não errar no que já conhecem, devendo estar prontas e preparadas ${ }^{22}$.

Das mulheres que relataram não ter dúvidas no período gestacional, a maioria possuía uma rede de apoio que a auxiliava frente às incertezas e/ou curiosidades.

A família é um dos componentes de auxílio de maior relevância e influência para o enfrentamento da gravidez. As pessoas mais próximas, como mãe e/ou responsável, companheiro, irmãs, sogra, tias, e outros, são consideradas uma das principais fontes de informações e apoio para as gestantes. No entanto, os familiares também possuem incertezas e anseios acerca da gestação que precisam ser sanados ${ }^{23}$.

0 uso da internet também foi considerado como uma acessível rede de apoio. Em relação a isto, é necessário que o profissional de saúde legitime fontes que sejam fidedignas e alerte para conteúdos sem base científica. Isso porque, comumente, em locais de busca da rede mundial de computadores, os sites que mais aparecem não são necessariamente os de conteúdo mais adequado, mas sim disseminadores de mitos ${ }^{24}$.

A Unidade de Saúde do bairro onde as gestantes residem também foi citada como rede de apoio. Este estabelecimento pode prover a implantação dos grupos de educação em saúde, possibilitar o diálogo, reflexões e a participação de todos; incluindo as famílias ${ }^{24}$. Quando a mulher possui o apoio social, a sua consciência e sensatez como mãe são melhoradas ${ }^{25}$. Todas essas fontes de apoio apresentadas sanam o desconhecimento das mulheres enquanto mães e orientam ações para manter o feto saudável na gravidez. Assim, ajudam a melhorar as condições de vida, acolhimento e segurança ${ }^{26}$.

Porém, mesmo ante esses amparos há fatores que poderiam melhorar ainda mais a experiência da gestação em relação às dúvidas, como a melhoria do serviço público de saúde e o acesso à educação. Sabe-se que a maior parte das dificuldades vivenciadas pelas gestantes podem ser resolvidas, por meio de ações de educação geral, treinamento, prevenção e educação específica em saúde ${ }^{23,25}$.

A criação de grupos de gestantes foi algo sugerido pelas pesquisadas. Este método de abordagem tem se revelado como estratégia de desenvolvimento do conhecimento, uma vez que os participantes contribuem com suas experiências e questionamentos. Nestes encontros, o profissional de saúde intermedia a reunião por meio do embasamento técnico-científico, gerando confiança. Na ausência desse profissional, a receptividade pode ser comprometida ${ }^{5,7}$.

Um ensaio clínico, controlado e randomizado, realizado na Califórnia/USA, evidenciou que o atendimento pré-natal em grupo é bem aceito pelas gestantes, que se mostraram 
satisfeitas com esse tipo de acompanhamento; além de impactar positivamente nos desfechos perinatais, como menor risco de parto prematuro ${ }^{27}$.

A maior valorização da "escuta ativa" traz abertura para a grávida expor suas preocupações, dando a ela atenção plena sobre o que realmente deseja expressar. Esta disposição não deverá ser restrita apenas à ação de ouvir, como também em interpretar e procurar compreender genuinamente a gestante tanto pela sua fala como pelo seu silêncio voluntário. 0 profissional deve estar pronto para lidar com as principais emoções das gestantes, tais como: confiança, alegria, tristeza, medo, raiva, surpresa, aversão e antecipação; tendo consciência de que a abertura e a empatia têm relevância maior que qualquer outro tipo de assistência ${ }^{19}$.

\section{CONCLUSÃo}

As dúvidas vivenciadas na gestação foram bastante variadas, englobando alterações fisiológicas, amamentação, parto e nascimento; até os cuidados com o recém-nascido e outras. Como redes de apoio, destacou-se os familiares, a internet e a UBS.

As limitações deste estudo referem-se a amostragem intencional de conveniência, o delineamento exploratório descritivo, bem como o quantitativo de participantes; o que dificulta a realização de análises estatísticas inferenciais. Contudo, foi compreensível que as dúvidas levantadas se mostraram presentes em todos os trimestres da gravidez, bem como entre primigestas e entre aquelas que já possuíam outras experiências gestacionais.

Acredita-se que esses achados possam auxiliar os profissionais de saúde que atuam no cuidado à gestante na atenção primária à saúde a ampliar suas ações de educação em saúde, com diferentes abordagens nas consultas de pré-natal para sanar as principais dúvidas das mulheres e assim proporcionar uma melhor experiência gestacional apresentando menos riscos à mãe e ao bebê.

Diante da carência de pesquisas e aprofundamento acerca da temática, sugere-se a realização de novos estudos com diferentes delineamentos metodológicos, a fim de explorar ainda mais essa temática tão relevante para a saúde materno-infantil.

\section{REFERÊNCIAS}

1. Hussein SAAA, Dahlen HG, Ogunsiji O, Schmied V. Jordanian women's experiences and constructions of labour and birth in different settings, over time and across generations: a qualitative study. BMC Pregnancy Childbirth [Internet]. 2020 [citado em 15 jun 2020]; 20(357):1-15. DOI: 10.1186/s12884-020-03034-3

2. Onyeze-Joe C, Godin I. Experiences, views and needs of first-time fathers in pregnancy-related care: a qualitative study in south-East Nigeria. BMC Pregnancy and Childbirth [Internet]. 2020 [citado em 10 jun 2020]; 20(213):1-11. DOI: 10.1186/s12884-020-02889-w

3. Magalhães SQC, Fracolli LA, Siqueira LD, Chiesa AM, Reticena K de O. Contributes of pre-natal to self-care of women assisted by family health teams. Ciênc Cuid Saúde [Internet]. 2018 [citado em 15 jul 2020];17(2):39994. DOI: 10.4025/cienccuidsaude.v17i2.39994

4. Hetherington E, McDonald S, Williamson T, Tough S. Trajectories of social support in pregnancy and early postpartum: findings from the All Our Families cohort. Soc Psychiatry Psychiatr Epidemiol [Internet]. 2020 [citado em 20 jun 2020]; 55:259-67. DOI: 10.1007/s00127-019-01740-8

5. Sampaio LM, Reis AP, Neves GAO, Andrade DL. Street network: importance of prenatal followup and linking of pregnant to reference maternity. Ciênc Cuid Saúde [Internet]. 2018 [citado em 20 maio 2020]; 17(1):38384. DOI: 10.4025/cienccuidsaude.v17i1.38384

6. Buser JM, Moyer CA, Boyd CJ, Zulu D, Ngoma-Hazemba A, Mtenje JT, et al. Maternal knowledge of essential newborn care in rural Zambia. Health Care Women Int. [Internet]. 2020 [citado em 15 ago 2020]; 42(4-6):778-93. DOI: 10.1080/07399332.2020.1781125 
7. Teixeira FV, Linhares AEP, Guimarães RX, Cavalcante MMB, Lopes AIN, Teixeira MA. Oficinas educativas para um grupo de gestantes acerca do período gravídico. SANARE [Internet]. 2016 [citado em 13 jun 2020]; 15(1):119-25. Disponível em: https://sanare.emnuvens.com.br/sanare/article/view/937

8. Januaria IS, Souza PF, Souza MAL, Takeshita IM. Nursing students in their work with pregnant women and the main doubts: experience report. REAS [Internet]. 2020 [citado em 27 ago 2020]; 51:e3467. DOI: 10.25248/reas.e3467.2020

9. Sayakhot P, Carolan-Olah M. Internet use by pregnant women seeking pregnancy-related information: a systematic review. BMC Pregnancy Childbirth [Internet]. 2016 [citado em 13 ago 2020]; 16(65):1-10. DOI: 10.1186/s12884-016-0856-5

10. Tostes NA, Seidl EMF. Expectativas de gestantes sobre o parto e suas percepções acerca da preparação para o parto. Temas Psicol. [Internet]. 2016 [citado em 09 jul 2020]; 24(2): 681-93. DOI: $10.9788 / \mathrm{TP} 2016.2-15$

11. Cabral SAAO, Carmo LA, Barbosa SES, Fontes NAS, Gabriel IS, Barroso ML. Conhecimento das gestantes acerca do parto na admissão intrapartal. Id on Line Rev Mult Psic [Internet]. 2018 [citado em 15 jun 2020]; 12(39):851-64. DOI: https://doi.org/10.14295/idonline.v12i39.1034 12. Taylor J, Hicks CW, Heller JA. The hemodynamic effects of pregnancy on the lower extremity venous system. J Vasc Surg. [Internet]. 2018 [citado em 25 ago 2020]; 6(2):246-55. DOI: 10.1016/j.jvsv.2017.08.001

13. Soma-Pillay P, Nelson-Piercy C, Tolppanen H, Mebazaa A. Physiological changes in pregnancy. Cardiovasc J Afr. [Internet]. 2016 [citado em 11 jul 2020]; 27(2):89-94. DOI: 10.5830/CVJA-2016-021

14. Oliveira DS, Boccolini CS, Faerstein E, Verly-Jr E. Duração do aleitamento materno e fatores associados entre 1960 e 2000. J Pediatr. [Internet]. 2017 [citado em 01 set 2020]; 93(2):130-5. DOI: $10.1016 / j . j p e d .2016 .05 .005$

15. Nunes MD, Rigo L, Boclin KLS, Malvestio LMM, Anziliero D, Horta BL, et al. Qualidade do PréNatal no Brasil: Pesquisa Nacional de Saúde 2013. Ciênc Saúde Colet. [Internet]. 2019 [citado em 12 jun 2020]; 24(3):1223-32. DOI: 10.1590/141381232018243.13122017

16. Viellas EF, Domingues RMSM, Dias MAB, Gama SGN, Theme-Filha MM, Costa JV, et al. Assistência pré-natal no Brasil. Cad Saúde Pública [Internet]. 2014 [citado em 15 ago 2020]; 30(Sup):S85-S100. DOI: 10.1590/0102-311X00126013

17. Cohen SS, Alexander DD, Krebs NF, Young BE, Cabana MD, Erdmann P, et al. Factors associated with breastfeeding initiation and continuation: a meta-analysis. J Pediatr. [Internet]. 2018 [citado em 11 ago 2021]; 203:190-6.e21. DOI: 10.1016/j.jpeds.2018.08.008

18. Silva ACL, Félix HCR, Ferreira MBG, Wysocki AD, Contim D, Ruiz MT. Preferência pelo tipo de parto, fatores associados à expectativa e satisfação com o parto. Rev Eletr Enf. [Internet]. 2017 [citado em 10 ago 2020]; 19:a34. DOI: 10.5216/ree.v19.44139

19. Tomasi E, Fernandes PAA, Fischer T, Siqueira FCV, Silveira DS, Thumé E, et al. Qualidade da atenção pré-natal na rede básica de saúde do Brasil: indicadores e desigualdades sociais. Cad Saúde Pública [Internet]. 2017 [citado em 07 jul 2020]; 33(3):e00195815. DOI: 10.1590/0102311x00195815

20. Vasconcelos ML, Pessoa VLMP, Chaves EMC, Pitombeira MGV, Moreira TMM, Cruz MR, et al. Cuidado à criança menor de seis meses no domicilio: experiência da mãe primípara. Esc Anna Nery Rev Enferm. [Internet]. 2019 [citado em 23 ago 2020]; 23(3):e20180175. DOI: 10.1590/2177-9465-ean-2018-0175

21. Xavier ACA, Jesus MGR, Pereira AS, Santos KA, Ferreira DAS, Sales SNV. Cuidados com o recém-nascido. Rev Inov, Tecnol Ciênc. 2017 [citado em 08 jun 2020]; 1(1):168-72.

22. Schiavo RA, Rodrigues OMPR, Perosa GB. Variables associated with gestational anxiety in primigravidas and multigravidas. Trends Psychol. [Internet]. 2018 [citado em 10 jun 2020]; 26(4):2091-104. DOI: 10.9788/TP2018.4-14Pt 
23. Tissot DW, Falcke D. Estrutura e dinâmica de funcionamento das famílias de origem de casal com gravidez na adolescência. Pensando Fam. [Internet]. 2018 [citado em 07 jun 2020]; 23(2):58-74. Disponível em: http://pepsic.bvsalud.org/pdf/penf/v23n2/v23n2a06.pdf

24. Silva DD, Schmitt IM, Costa R, Zampieri MFM, Bohn IE, Lima MM. Promoção do aleitamento materno no pré-natal: discurso das gestantes e dos profissionais de saúde. REME Rev Min Enferm. [Internet]. 2018 [citado em 05 jun 2020]; 22:e-1103. DOI: 10.5935/14152762.20180031

25. Manente MV, Rodrigues OMPR. Maternidade e trabalho: associação entre depressão pósparto, apoio social e satisfação conjugal. Pensando Fam. [Internet]. 2016 [citado em 19 jun 2020]; 20(1):99-111. http://pepsic.bvsalud.org/pdf/penf/v20n1/v20n1a08.pdf

Disponível

em:

26. Matos GC, Soares MR, Escobal APL, Quadro PP, Rodrigues JB. Rede de apoio familiar à gravidez e ao parto na adolescência: uma abordagem moscoviciana. J Nurs Health [Internet]. 2019 [citado em 28 jun 2020]; 9(1):1-9. DOI: 10.15210/JONAH.V9I1.12754

27. Tubay AT, Mansalis KA, Simpson MJ, Armitage NH, Briscoe G, Potts V. The effects of group prenatal care on infant birthweight and maternal well-being: a randomized controlled trial. Mil Med. [Internet]. 2019 [citado em 11 ago 2021]; 184(5-6):e440-e446. DOI: $10.1093 /$ milmed/usy361

Editora Associada: Vania Del Arco Paschoal

\section{CONTRIBUIÇÕES}

Beatriz Lisboa de Macedo Brito contribuiu na concepção, coleta e análise dos dados, redação e revisão. Bianca Machado Cruz Shibukawa, Ieda Harumi Higarashi, Marcela Demitto Furtado e Maria de Fátima Garcia Lopes Merino participaram da redação e revisão.

\section{Como citar este artigo (Vancouver)}

Brito BLM, Shibukawa BMC, Merino MFGL, Higarashi IH, Furtado MD. Dúvidas na gestação: vivência de mulheres acompanhadas na atenção primária à saúde. REFACS [Internet]. 2021 [citado em inserir dia, mês e ano de acesso]; 9(4):903-11. Disponível em: inserir link de acesso. DOI: inserir link do DOI.

\section{Como citar este artigo (ABNT)}

BRITO, B. L. de M.; SHIBUKAWA, B. M. C.; MERINO, M. de F. G. L.; HIGARASHI, I. H.; FURTADO, M. D. Dúvidas na gestação: vivência de mulheres acompanhadas na atenção primária à saúde. REFACS, Uberaba, MG, v. 9, n. 4, p. 903-11, 2021. Disponível em: inserir link de acesso. Acesso em: inserir dia, mês e ano de acesso. DOI: inserir link do DOI.

\section{Como citar este artigo (APA)}

Brito, B.L.M., Shibukawa, B.M.C., Merino, M.F.G.L., Higarashi, I.H., \& Furtado, M.D. (2021). Dúvidas na gestação: vivência de mulheres acompanhadas na atenção primária à saúde. REFACS, 9(4), 903-11. Recuperado em: inserir dia, mês e ano de acesso de inserir link de acesso. DOI: inserir link do DOI. 